Article

\title{
Surface State Dynamics of Topological Insulators Investigated by Femtosecond Time- and Angle-Resolved Photoemission Spectroscopy
}

\author{
Hamoon Hedayat ${ }^{1}$ (D), Davide Bugini ${ }^{1}$, Hemian $\mathrm{Yi}^{2}{ }^{2}$, Chaoyu Chen ${ }^{2}$, Xingjiang Zhou ${ }^{2}$, \\ Giulio Cerullo ${ }^{1,3}$ (D), Claudia Dallera ${ }^{1}$ and Ettore Carpene ${ }^{3, *}$ \\ 1 Dipartimento di Fisica, Politecnico di Milano, 20133 Milan, Italy; hamoon.hedayat@polimi.i (H.H.); \\ davide.bugini@polimi.it (D.B.); giulio.cerullo@polimi.it (G.C.); claudia.dallera@polimi.it (C.D.) \\ 2 National Lab for Superconductivity, Institute of Physics, Chinese Academy of Science, Beijing 100190, China; \\ hemian.yi@synchrotron-soleil.fr (H.Y.); csuccy@163.com (C.C.); XJZhou@aphy.iphy.ac.cn (X.Z.) \\ 3 IFN-CNR c/o Dipartimento di Fisica, Politecnico di Milano, 20133 Milan, Italy \\ * Correspondence: ettore.carpene@polimi.it
}

Received: 11 April 2018; Accepted: 27 April 2018; Published: 30 April 2018 updates

\begin{abstract}
Topological insulators (TI) are known for striking quantum phenomena associated with their spin-polarized topological surface state (TSS). The latter in particular forms a Dirac cone that bridges the energy gap between valence and conduction bands, providing a unique opportunity for prospective device applications. In TI of the $\mathrm{Bi}_{x} \mathrm{Sb}_{2-x} \mathrm{Te}_{y} \mathrm{Se}_{3-y}$ (BSTS) family, stoichiometry determines the morphology and position of the Dirac cone with respect to the Fermi level. In order to engineer specific transport properties, a careful tuning of the TSS is highly desired. Therefore, we have systematically explored BSTS samples with different stoichiometries by time- and angle-resolved photoemission spectroscopy (TARPES). This technique provides snapshots of the electronic structure and discloses the carrier dynamics in surface and bulk states, providing crucial information for the design of electro-spin current devices. Our results reveal the central role of doping level on the Dirac cone structure and its femtosecond dynamics. In particular, an extraordinarily long TSS lifetime is observed when the the vertex of the Dirac cone lies at the Fermi level.
\end{abstract}

Keywords: TARPES; topological insulator; electronic dynamics; topological surface state; BSTS; ultrafast spectroscopy; Dirac cone

\section{Introduction}

Due to their unconventional electronic structure, topological insulators (TI) are potential candidates for next-generation electronic and spintronic devices [1-3]. They behave like standard insulators or semiconductors in the bulk, but stimulating physics takes place at their surface. Here, the fermion gas forms a conductive state with a Dirac cone structure in the Brillouin zone and a helical spin texture. Recent studies have shown several unique features of TI, including observations of the quantum spin Hall effect [4], Majorana fermions [5], the topological magnetoelectric effect [6], magnetic monopoles [7], nonlinear modulation of the electromagnetic field [8], and the anomalous quantum Hall effect [9]. In practice, some difficulties have prevented the widespread use of TI in mainstream electronic components. The major obstacle is the large contribution of bulk electrons that hamper surface transport properties [10]. It has been shown that a reduction of bulk effects leads to better control of the interesting surface phenomena. In particular, focusing on the $\mathrm{Bi}_{x} \mathrm{Sb}_{2-x} \mathrm{Te}_{y} \mathrm{Se}_{3-y}$ (BSTS) family, some of the most promising ternary and quaternary compounds were identified as $\mathrm{Bi}_{2} \mathrm{Te}_{2} \mathrm{Se}$ [11], $\mathrm{Bi}_{x} \mathrm{Sb}_{x-2} \mathrm{Te}_{3}$ [12], $\mathrm{Bi}_{0.5} \mathrm{Sb}_{1.5} \mathrm{Te}_{1.8} \mathrm{Se}_{1.2}$ [13] and BiSbTeSe 2 [14]. These investigations have 
shown that fine-tuning the BSTS stoichiometry allows one to control the Dirac cone structure and, consequently, the electronic and spin properties of the TI [15-18].

From the experimental point of view, angle-resolved photoemission spectroscopy (ARPES) has been a key tool to disclose the main features of TI, as it provides a direct picture of the occupied electronic states in the Brillouin zone [19-21]. Being conductive, the topological surface states (TSS) are only partially filled. However, complete knowledge of their structure would be highly desirable, especially if one wishes to tailor specific charge and spin transport properties. To overcome this limitation an advanced approach combines ARPES and pump-probe techniques, resulting in the so-called time-resolved ARPES (TARPES). Here, the sample is perturbed by a short laser pulse-the pump-which promotes electrons to unoccupied states above the Fermi level. Recording ARPES images during the excitation and relaxation processes, one can access the electronic structure of both occupied and empty states, and also reveal the main electronic scattering processes as thermodynamic equilibrium is restored after photoexcitation [22-26].

In this paper, we investigate the ultrafast electronic dynamics of selected BSTS samples by means of TARPES with sub-100 femtosecond time resolution. The binding energy of the Dirac node (DN), i.e., the energy where upper and lower branches of the Dirac cone meet, can be tuned from $-0.3 \mathrm{eV}$ to $+0.2 \mathrm{eV}$, depending on the BSTS stoichiometry. The electron density of states (DOS) is drastically reduced in the vicinity of the $\mathrm{DN}$, leading to a strong alteration of electronic interactions [27]. Therefore, BSTS samples offer an unprecedented opportunity to investigate the electronic behavior of the TSS in relation to its filling, i.e., to the position of the DN with respect to the Fermi level. Real applications require long-lasting electronic dynamics at a TI's surface (which usually only last a few picoseconds). Our results show that Dirac carriers relax on an extraordinarily long timescale when the binding energy of the $\mathrm{DN}$ is vanishingly small. We believe this novel observation might provide critical information to guide the fabrication of innovative TI-based spintronic devices.

\section{Materials and Methods}

$\mathrm{Bi}_{x} \mathrm{Sb}_{2-x} \mathrm{Te}_{y} \mathrm{Se}_{3-y}$ (BSTS) single crystals were grown by a self-flux method. The high-purity raw materials were weighed and mixed according to the desired stoichiometry. The specimens were heated up to $1000{ }^{\circ} \mathrm{C}$ for $12 \mathrm{~h}$, then slowly cooled down to $500^{\circ} \mathrm{C}$ for over $100 \mathrm{~h}$, before finally being cooled to room temperature. Single crystals of several millimeters in size were obtained (see Reference [28] for more details). Here, we focus on the following compounds: $\mathrm{Sb}_{2} \mathrm{Te}_{3}, \mathrm{Bi}_{0.5} \mathrm{Sb}_{1.5} \mathrm{TeSe}_{2}, \mathrm{Bi}_{0.8} \mathrm{Sb}_{1.2} \mathrm{Te}_{3}$, $\mathrm{Bi}_{2} \mathrm{Te}_{3}, \mathrm{Bi}_{2} \mathrm{Te}_{2.65} \mathrm{Se}_{0.35}$, and $\mathrm{Bi}_{2} \mathrm{Te}_{0.5} \mathrm{Se}_{2.5}$.

Figure 1 schematically illustrates our TARPES experimental apparatus. The laser source is a commercial Yb-based system (Pharos, Light Conversion) generating 300 fs pulses with photon energy $h v=1.2 \mathrm{eV}$ (wavelength $\lambda=1030 \mathrm{~nm}$ ) at $100 \mathrm{kHz}$ repetition rate (for details about the optical system see Reference [29]). The laser feeds a home-built non-collinear optical parametric amplifier (NOPA) that provides ultrashort pump photons of energy $h v=1.85 \mathrm{eV}(\lambda=670 \mathrm{~nm})$ with a pulse duration of $30 \mathrm{fs}$. A sum frequency generation (SFG) stage generates UV probe photons with energy $h v=6 \mathrm{eV}$ $(\lambda=206 \mathrm{~nm})$ and a pulse duration of $65 \mathrm{fs}$. The instrumental response function (IRF) given by the cross-correlation of pump and probe pulses, thus corresponds to an overall time resolution of about 72 fs. Pump and probe beams, focused respectively on spots of about $100 \mu \mathrm{m}$ and $50 \mu \mathrm{m}$ in diameter, converge on a sample placed inside an ultrahigh vacuum chamber (pressure $\approx 10^{-10} \mathrm{mbar}$ ) and cleaved in situ before each measurement.

Photo-emitted electrons are detected by a home-built time of flight (ToF) spectrometer equipped with a micro channel plate (MCP). Rotation of the sample's normal with respect to the analyzer axis provides the angular resolution (for more details of the ToF analyzer and acquisition system see Reference [30]). All spectra were recorded at room temperature. 


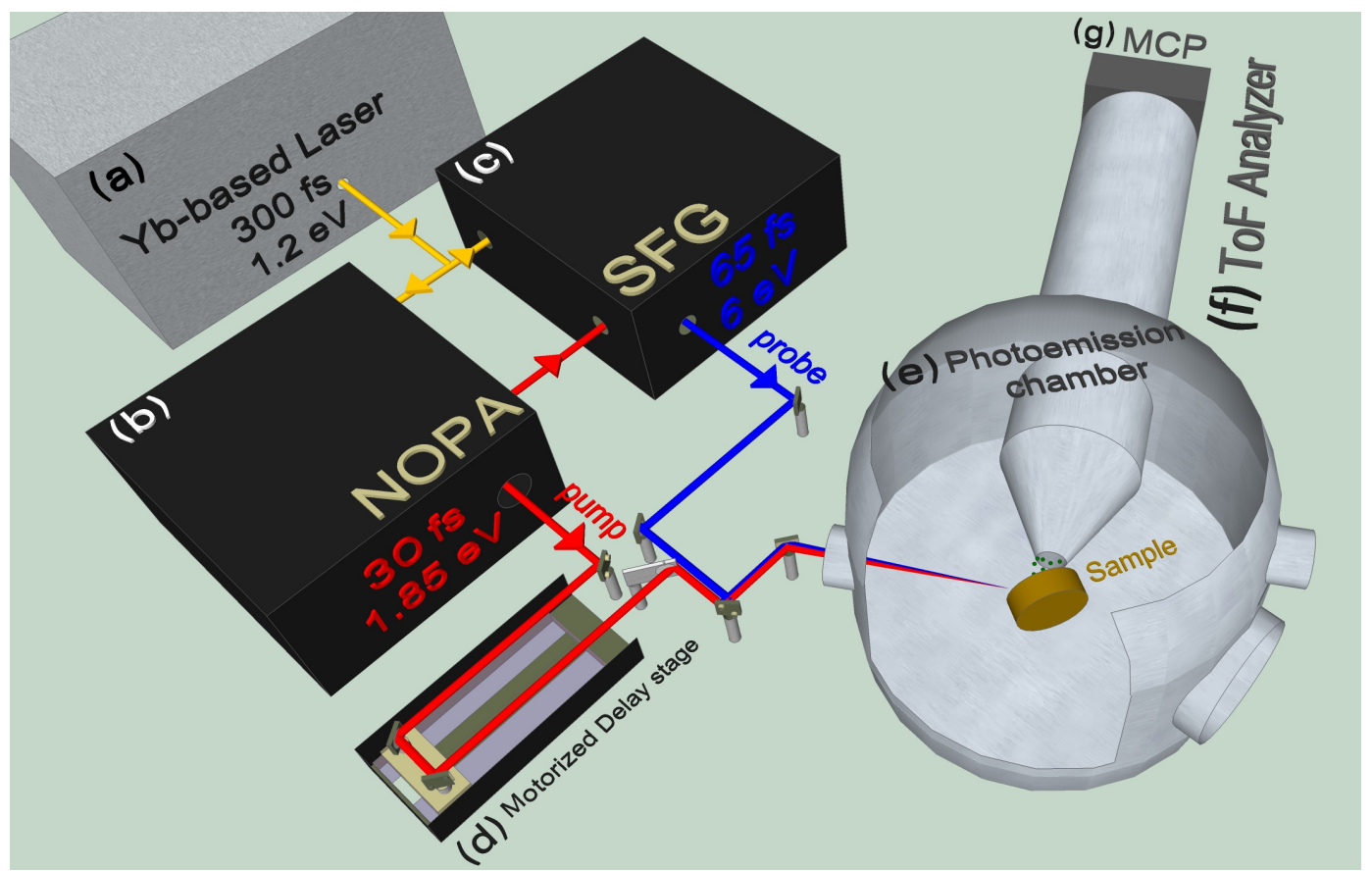

Figure 1. Schematic drawing of a time- and angle-resolved photoemission spectroscopy (TARPES) system: (a) Yb-based laser system generating 300 fs laser pulses with photon energy $h v=1.2 \mathrm{eV}$ $(\lambda=1030 \mathrm{~nm})$ at repetition rate of $100 \mathrm{kHz}$; (b) non-collinear optical parametric amplifier (NOPA) and (c) sum frequency generation (SFG) stage, which produce the pump (hv=1.85 eV, $30 \mathrm{fs})$ and probe ( $\mathrm{h} v=6 \mathrm{eV}, 65 \mathrm{fs}$ ) beams, respectively; (d) pump delay stage; (e) ultrahigh vacuum chamber; (f) time-of-flight (ToF) analyzer equipped with a (g) micro channel plate (MCP) detector [29].

\section{Results and Discussion}

Figure 2 reports TARPES measurements of selected BSTS samples. Each panel shows the spectra at three illustrative pump-probe delays: before pump arrival (left, "-" label), at zero delay (center, "0" label) and one picosecond after pumping (right, " + " label). The linear dispersion of the TSS is clearly observed for all compounds and marked with dashed lines to guide the eye. Moving from panel (a) to panel (f) in Figure 2, the amounts of Bi and Se increase at the expenses of Sb and Te and the samples become progressively n-doped. The Dirac cone of the most p-doped sample, $\mathrm{Sb}_{2} \mathrm{Te}_{3}$ in panel (a), is unoccupied at negative delays. Upon pumping, electrons are optically transferred above the Fermi energy (FE), filling the TSS. At the opposite extreme, the most n-doped sample, $\mathrm{Bi}_{2} \mathrm{Te}_{0.5} \mathrm{Se}_{2.5}$ in panel (f), has an almost completely occupied TSS even under equilibrium conditions (negative delay). It should be noticed that the $\mathrm{DN}$ of the $\mathrm{Bi}_{0.8} \mathrm{Sb}_{1.2} \mathrm{Te}_{3}$ sample, panel (c), has vanishing binding energy. As we will see in the next section, this feature has important consequences on the dynamics of Dirac fermions. We also point out that, regardless of the stoichiometry, at zero pump-probe delay two bulk bands (named BB1 and BB2 in Figure 2) are transiently populated, while after one picosecond most of the excess spectral weight induced by photoexcitation has been transferred from BB1 (higher energy) to BB2 (lower energy) and the TSS. 

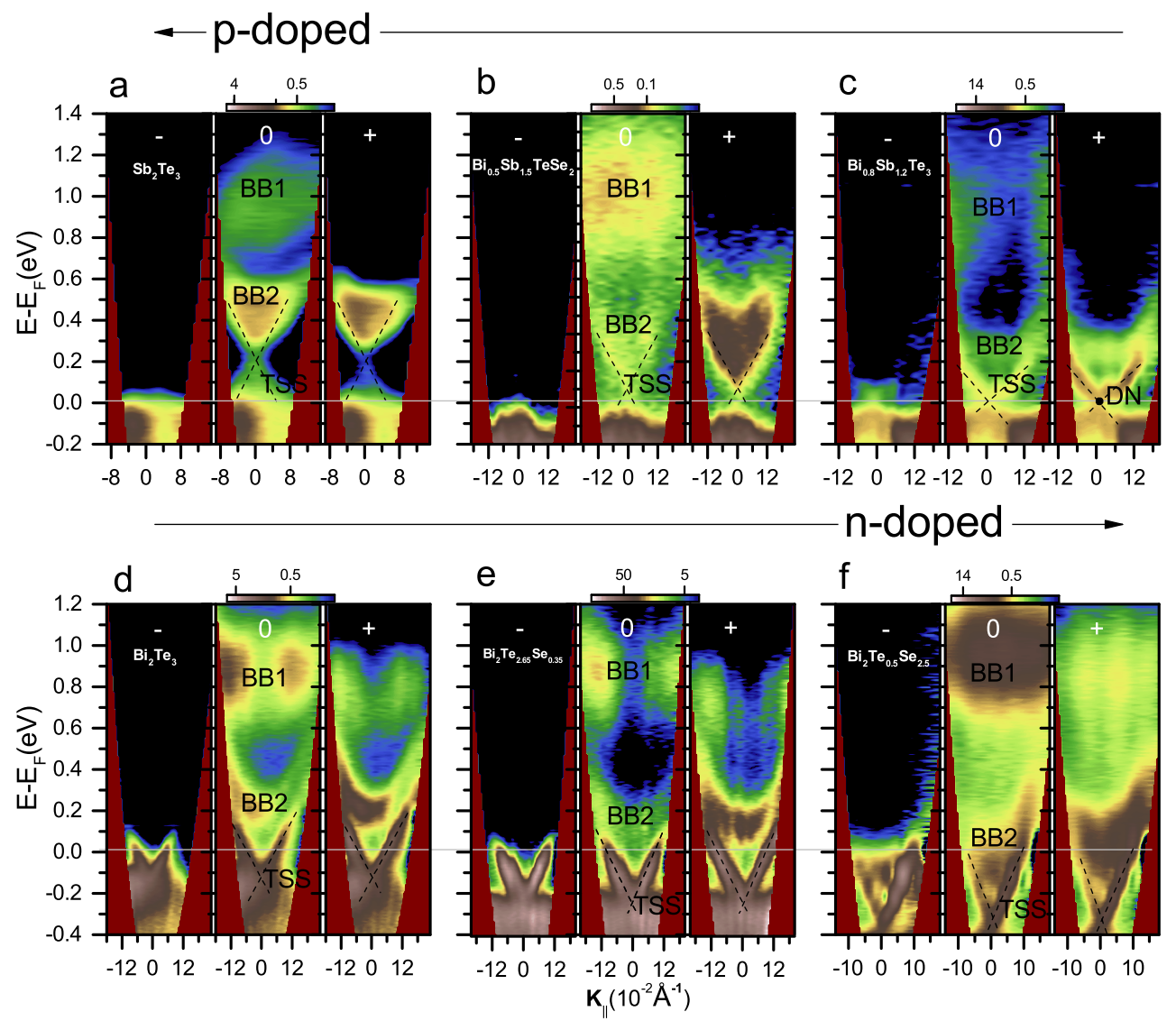

Figure 2. TARPES snapshots of six BSTS samples: (a) $\mathrm{Sb}_{2} \mathrm{Te}_{3}$, (b) $\mathrm{Bi}_{0.5} \mathrm{Sb}_{1.5} \mathrm{TeSe}_{2}$, (c) $\mathrm{Bi}_{0.8} \mathrm{Sb}_{1.2} \mathrm{Te}_{3}$, (d) $\mathrm{Bi}_{2} \mathrm{Te}_{3}$, (e) $\mathrm{Bi}_{2} \mathrm{Te}_{2.65} \mathrm{Se}_{0.35}$, and (f) $\mathrm{Bi}_{2} \mathrm{Te}_{0.5} \mathrm{Se}_{2.5}$. For each sample three different pump-probe delays are shown: negative ("-" label, left) i.e., the equilibrium condition, zero ("0" label, center), +1 ps ("+" label, right). Fermi level is shown by the gray horizontal lines. Dashed black lines are guides to the eye, showing the topological surface states (TSS). Bulk bands (BB1 and BB2) are clearly visible at zero delay. Passing from panel (a) to panel (f) the samples are progressively n-doped. Color intensity maps are on a logarithmic scale.

In order to better visualize the photo-excited band structure and dynamics reported in Figure 2, we have subtracted the equilibrium TARPES maps (panels marked with negative sign) from the pumped spectra (marked with zero and positive sign). The results are plotted in Figure 3 (same sample sequence as Figure 2). The upper and lower rows refer to the band structure at zero and at $+1 \mathrm{ps}$ delays, respectively. Photo-induced electronic population is represented in red, while band depletion is displayed in green. The large gray arrows connect all $\mathrm{DN}$, revealing a regular $100 \mathrm{meV}$ decrease of the TSS binding energy for adjacent samples. It is also interesting to observe that in the samples where the DN lies above the Fermi level (panels a and b), even one picosecond past the optical excitation (bottom row) the lower branch of the Dirac cone is not significantly populated. This dynamical behavior has been previously observed in $\mathrm{Sb}_{2} \mathrm{Te}_{3}$ [27], but seems to be a rather general feature due to the so-called bottleneck effect related to the vanishing phase space at the DN.

The electronic dynamics of each band (BB1, BB2, and TSS) were extracted by integrating the spectral intensity within the small energy-momentum boxes shown in the upper row in Figure 3: boxes 1 and 2 refer to BB1 and BB2, respectively, while boxes 3 and 4 refer to the TSS. The results are reported in Figure 4. We have followed the same color scheme used for the boxes in Figure 3: as the $\mathrm{DN}$ decreases in energy (i.e., p- to n-doping), the line color turns lighter. All curves are normalized to their respective maxima. 


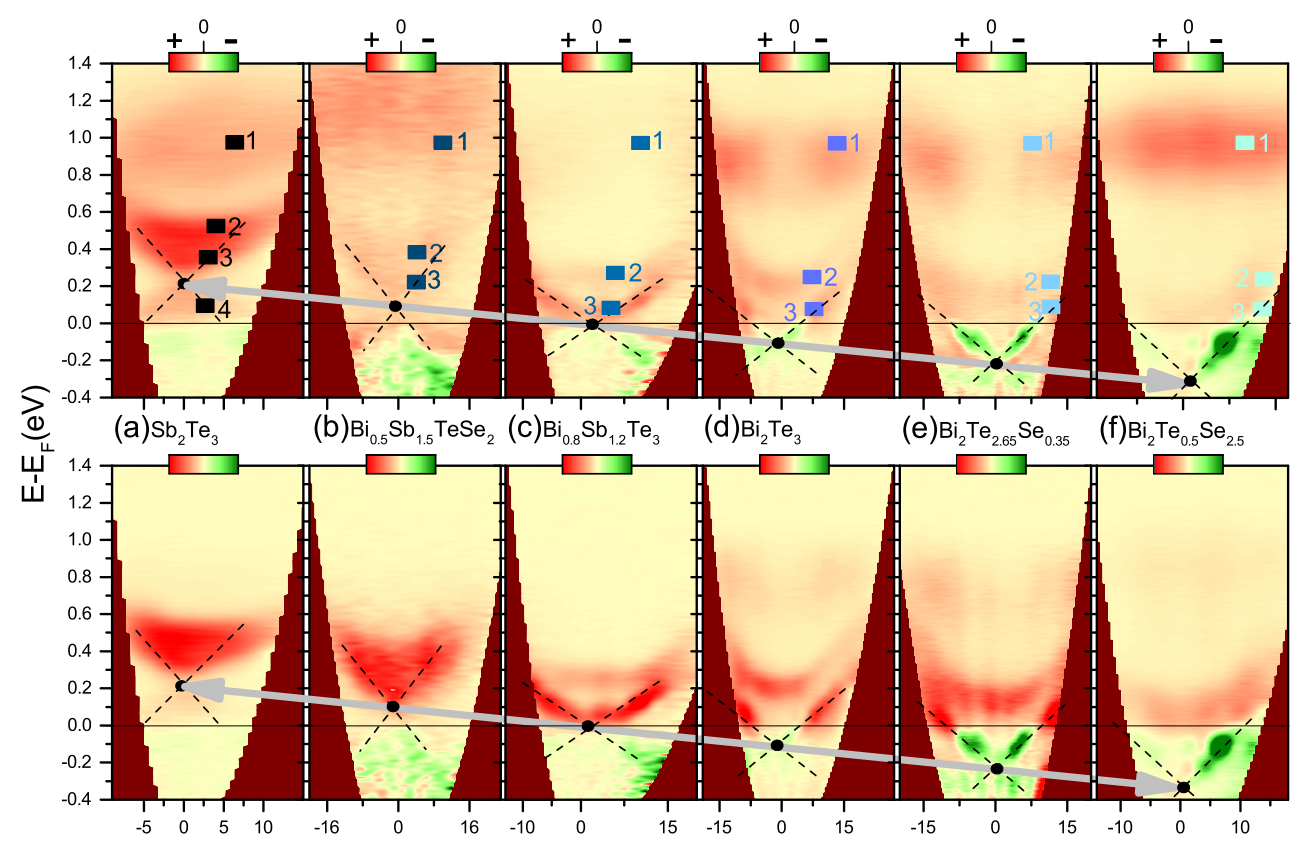

Figure 3. Photo-induced electronic population (red) and depletion (green) of the BSTS samples at 0 delay (top row) and 1 ps past the pump (bottom row). Same sample order of Figure 2 from (a) to (f). The black dashed lines highlight the TSS. The thick gray arrows connect the Dirac nodes (DN) of the BSTS compounds. The small boxes labeled 1 to 4 show the integration windows used to estimate the temporal dynamics on the different bands. The box color becomes lighter as the sample passes from p-doped (panel a) to n-doped (panel f).

Figure 4a shows the dynamics of BB1 (the high-energy bulk band). Within experimental noise all curves are identical and can be fit by a simple phenomenological function: an exponential decay convoluted with the IRF of the instrument (red dashed line). This fit is reproduced in all other panels (b-f) for comparison. Previous investigations reported similar fast dynamics of BB1 [31]. Here we notice that, upon pumping, electrons are optically transferred to BB1 states and subsequently relax to lower energy states with a characteristic time of $220 \mathrm{fs}$, regardless of the doping level. Therefore, bulk relaxation seems to be unaffected by sample doping and in particular by the filling degree of the TSS. Moreover, in all analyzed BSTS samples, the BB2 state (the low-energy bulk band) is largely unoccupied before pumping and it is only mildly photo-populated. Thus, it can easily accommodate most of the electrons decaying from higher energy levels. According to this observation, it seems that BB1-to-BB2 is the dominant interband scattering process leading to the electronic relaxation of BB1 states. In other words, the TSS does not significantly affect the BB1 dynamics.

On the other hand, there seems to be an interplay between BB2 and TSS. Their electronic dynamics are shown in panels $(\mathrm{b}-\mathrm{c})$ of Figure 4. Several relaxation mechanisms have been proposed in the past, including electron-phonon interactions [22,31], spin-mediated channels [32], bulk diffusion [18], the photovoltage effect [23,33] and surface resonant states [34]. By comparing Figure $4 b$, the BB2, and Figure 4c, the TSS, on the same time scale we can clearly see that: (i) both relaxation dynamics are sensibly slower than BB1; (ii) there is a clear intensity build-up for p-doped samples (i.e., darker lines) (Figure $4 \mathrm{~b}$ ); and (iii) the slowest relaxation, especially for the TSS, takes place when the DN is at the Fermi level (i.e., the $\mathrm{Bi}_{0.8} \mathrm{Sb}_{1.2} \mathrm{Te}_{3}$ sample). These three considerations point out the similarities, and thus suggest a possible entanglement, between the dynamics of BB2 and TSS. In fact, some authors have already pointed out that BB2 might have a strong surface character, sharing the spin texture of the TSS $[34,35]$. This aspect is beyond the scope of the present study, but each of the three previous points deserve some comments. 


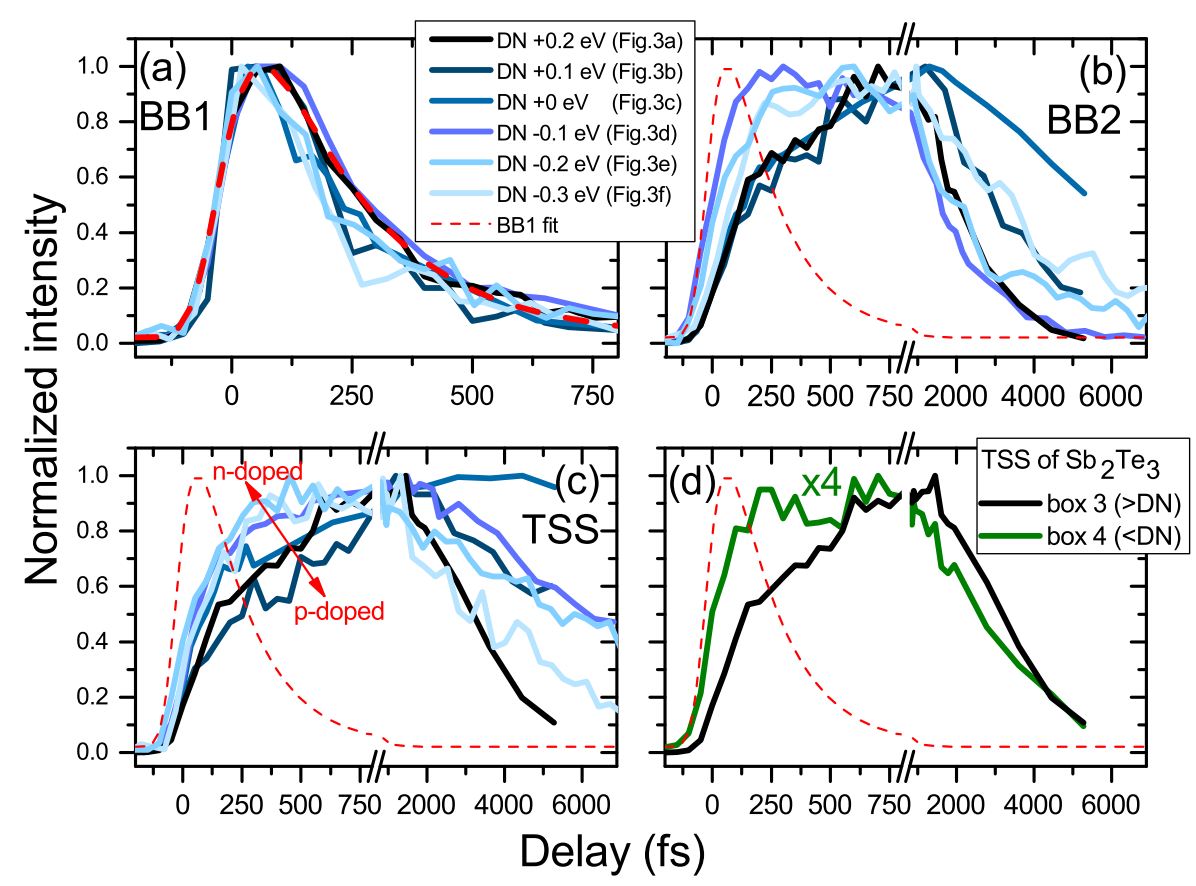

Figure 4. Summary of the electronic dynamics for different bands and doping levels (same color code as Figure 3) of the BSTS samples. The curves are normalized to their respective maxima. (a) BB1 band dynamics (box 1 in Figure 3). The red dashed line is a phenomenological fit and it has been reproduced in all panels for comparison. (b) BB2 band dynamics (box 2 in Figure 3). (c) TSS band dynamics (box 3 in Figure 3). The intensity build-up is faster for p-doped samples (as evidenced by the red arrow). Notice the extremely long electron lifetime for the undoped samples (DN at the Fermi level). (d) Comparative dynamics of the upper and lower branches of the TSS in $\mathrm{Sb}_{2} \mathrm{Te}_{3}$ (boxes 3 and 4 of Figure 3a).

Point (i) is probably the most trivial. The fact that relaxation times in BB2 and TSS are longer than in BB1 can be simply understood in terms of Fermi's golden rule: the higher the energy of the excited particle, the larger the number of available (empty) states it can decay into, and consequently a larger decay rate (i.e., faster decay time).

Point (ii) can be interpreted in terms of intra-band scattering processes. Referring to Figure 3, the p-doped samples (panels a and b) have their DN well above the Fermi level. Thus, both the BB2 and the upper branch of the TSS have unoccupied band minima at $\Gamma$ (the center of the Brillouin zone) under equilibrium conditions. As photo-excited electrons decay on BB2 and TSS states from higher levels, they accumulate towards the respective band minima through intra-band scattering. This process happens on a timescale that is clearly longer than the BB1 relaxation and it is particularly critical for the TSS since the DN corresponds to a vanishing DOS, i.e., fewer and fewer electrons can be accommodated as the DN is approached. On the other hand, for $n$-doped samples (panels d, e, and $f$ of Figure 3) the band minimum lies below the Fermi level and the unoccupied portions of BB2 and TSS bands are away from $\Gamma$. In this case, the intensity build-up time is compatible with the BB1 relaxation time (compare lighter curves of panels a and c in Figure 4). As electrons decay from BB1 to BB2 and TSS, they rapidly disperse into the Fermi sea since they cannot accumulate on any band minimum.

We also point out that, according to our analysis, we have no evidence of direct, photo-induced population of BB2 and the upper branch of TSS. However, in Figure $4 \mathrm{~d}$ we compare the electronic dynamics of the upper and lower branches of the TSS in $\mathrm{Sb}_{2} \mathrm{Te}_{3}$ (boxes 3 and 4 of Figure $3 \mathrm{a}$, respectively). Although relaxation times are quite similar, the lower branch of the TSS shows a faster intensity build-up and a reduced spectral weight (about 4 times lower) compared to the higher branch. The faster build-up is in agreement with the absence of a band minimum (actually in the lower branch there is a maximum), while the reduced spectral weight is in part attributed to the lack of electron accumulation 
and in part the consequence of the bottleneck effect: intra-band scattering from the higher branch of the cone is hindered by the vanishing DOS at the Dirac node.

Point (iii)—the extremely long lifetime of TSS electrons when the DN is at the Fermi level—is the most intriguing feature. Figure 5 summarizes the analysis of the TSS dynamics. Panel (a) reports a typical phenomenological fit (the data refer to the $\mathrm{Sb}_{2} \mathrm{Te}_{3}$ sample): an exponential growth with rise time $\tau_{r}$ followed by an exponential decay with characteristic time $\tau_{d}$. The fitting function has been convoluted with the Gaussian IRF of our experimental setup.
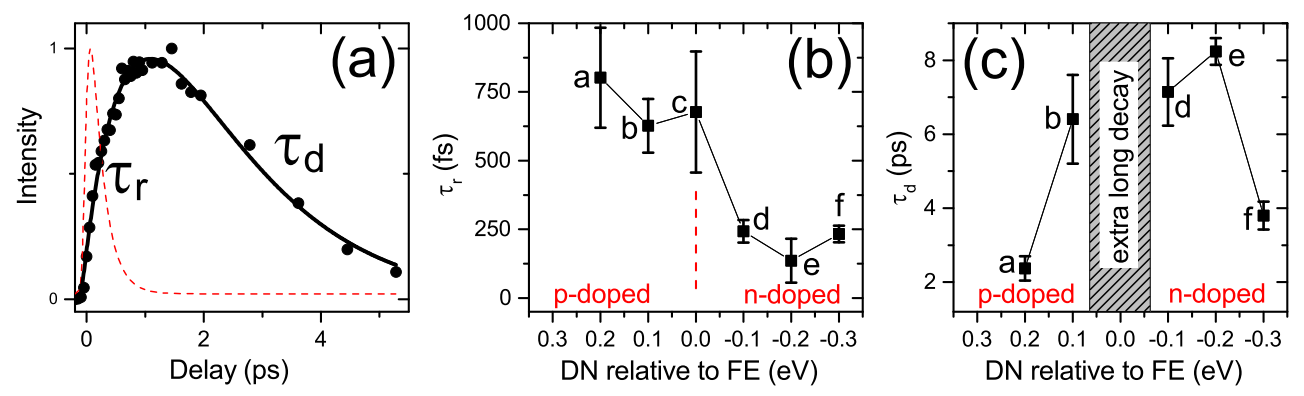

Figure 5. (a) Typical phenomenological fit of TSS electronic dynamics (data of $\mathrm{Sb}_{2} \mathrm{Te}_{3}$ sample): exponential growth with rise-time $\tau_{r}$, followed by an exponential decay with time constant $\tau_{d}$. The function is convoluted with the TARPES response time of $\approx 80 \mathrm{fs}$. TSS rise times (b) and decay times (c) of the different BSTS samples as a function of the DN energy relative to the Fermi level. The extra-long decay dynamics of $\mathrm{Bi}_{0.8} \mathrm{Sb}_{1.2} \mathrm{Te}_{3}(\mathrm{DN}=0 \mathrm{eV})$ cannot be fit within our experimental time window and the corresponding region is marked by the dashed area.

The obtained values of the time constants $\tau_{r}$ and $\tau_{d}$ are reported in panels (b) and (c), respectively, as a function of the DN binding energy (i.e., doping). As already discussed, the data of panel (b) show that $\mathrm{p}$-doped samples are characterized by a longer rise time (i.e., longer build-up) compared to the n-doped ones. And the latter nicely matches the decay time of BB1 states (220 fs, see Figure 4a). Figure $5 \mathrm{c}$ shows the decay time constants vs. doping. First, we point out that the higher the doping level (regardless of " $\mathrm{n}$ " or " $\mathrm{p}$ "), the faster the electronic decay. This behavior is most likely related to bulk contribution in the relaxation process (bulk diffusion according to Reference [18]). In fact, when the sample is highly p-doped (n-doped), the bulk valence (bulk conduction) band might intercept the Fermi level, providing additional states that could enhance the relaxation process, in the spirit of Fermi's golden rule. Therefore, a more isolated (i.e., bulk-free) TSS shows a longer decay. However, when the DN is at the the Fermi level, an exceptionally long electron persistence is observed in the TSS. This is the case of the $\mathrm{Bi}_{0.8} \mathrm{Sb}_{1.2} \mathrm{Te}_{3}$ sample (see the corresponding dark cyan curve in Figure $4 \mathrm{c}$ ), where the relaxation time is much longer than the investigated time windows (i.e., $\gg 7 \mathrm{ps}$ ). In this particular case, not only the absence of bulk contributions, but also the vanishing DOS in the TSS as the FE is approached is important. Under equilibrium conditions, the bulk conduction bands and the upper TTS branch are empty, while the bulk valence bands and the lower TSS branch are fully occupied. Upon laser pumping, electrons are excited to BB1 and subsequently decay to lower energy states. Since bulk diffusion is strongly hindered, electrons eventually accumulate on the lowest available energy levels that is the upper branch of the TSS. The extremely limited number of available states as the Fermi level is approached (keeping in mind that the DOS at the Fermi level is vanishingly small), leads to the extraordinarily long fermions lifetime, which can be interpreted as a strongly enhanced bottleneck effect.

It should be mentioned that some authors have claimed an additional effect that could increase the electronic lifetime in TSS; a photo-induced surface band bending (also known as surface photovoltage effect) $[23,33]$. This occurs when the TSS is well isolated from bulk states at the FE and leads to a different transient chemical potential of electrons and holes which creates a barrier for relaxation. However, its relevance to the observed enhancement of the electronic lifetime in TSS is still debated [36]. 


\section{Conclusions}

We have studied the electronic dynamics of the TSS in the BSTS family of compounds with different doping levels. We have focused on the relative position of the Dirac node (DN) with respect to the Fermi level. In particular, the close-to-intrinsic TI, where the DN is at the Fermi energy, shows a reduced effect of bulk states on the electronic properties of the TSS with an extraordinarily long lifetime of Dirac fermions. Our investigation reveals that TSS can be efficiently manipulated, providing an excellent platform to novel devices based on topological insulators.

Author Contributions: H.H., D.B., G.C., C.D., and E.C. conducted the TARPES experiments and analyzed the data. H.Y., C.C., and X.Z. prepared the BSTS samples. All authors contributed to this study and reviewed the manuscript.

Acknowledgments: This research is supported by Fondazione Cariplo (grant 2011-0389) and by Politecnico di Milano via the Interdepartmental Laboratory UPHOS. G.C. acknowledges support from the European Union Horizon 2020 Programme under grant agreement no. 696656. H.H. acknowledges financial support through the Postodctoral International Fellowship program of the Politecnico di Milano.

Conflicts of Interest: The authors declare no conflict of interest.

\section{References}

1. Moore, J.E. The birth of topological insulators. Nature 2010, 464, 194-198. [CrossRef] [PubMed]

2. Hsieh, D.; Qian, D.; Wray, L.; Xia, Y.; Hor, Y.S.; Cava, R.J.; Hasan, M.Z. A topological Dirac insulator in a quantum spin Hall phase. Nature 2008, 452, 970-974. [CrossRef] [PubMed]

3. Hsieh, D.; Xia, Y.; Wray, L.; Qian, D.; Pal, A.; Dil, J.; Osterwalder, J.; Meier, F.; Bihlmayer, G.; Kane, C.; et al. Observation of unconventional quantum spin textures in topological insulators. Science 2009, 323, 919-922. [CrossRef] [PubMed]

4. Qi, X.L.; Hughes, T.L.; Zhang, S.C. Fractional charge and quantized current in the quantum spin Hall state. Nat. Phys. 2008, 4, 273-276. [CrossRef]

5. Fu, L.; Kane, C.L. Superconducting proximity effect and Majorana fermions at the surface of a topological insulator. Phys. Rev. Lett. 2008, 100, 096407. [CrossRef] [PubMed]

6. Essin, A.M.; Moore, J.E.; Vanderbilt, D. Magnetoelectric polarizability and axion electrodynamics in crystalline insulators. Phys. Rev. Lett. 2009, 102, 146805. [CrossRef] [PubMed]

7. Qi, X.L.; Li, R.; Zang, J.; Zhang, S.C. Inducing a magnetic monopole with topological surface states. Science 2009, 323, 1184-1187. [CrossRef] [PubMed]

8. Li, R.; Wang, J.; Qi, X.L.; Zhang, S.C. Dynamical axion field in topological magnetic insulators. Nat. Phys. 2010, 6, 284-288. [CrossRef]

9. Yu, R.; Zhang, W.; Zhang, H.J.; Zhang, S.C.; Dai, X.; Fang, Z. Quantized anomalous Hall effect in magnetic topological insulators. Science 2010, 329, 61-64. [CrossRef] [PubMed]

10. Butch, N.P.; Kirshenbaum, K.; Syers, P.; Sushkov, A.B.; Jenkins, G.S.; Drew, H.D.; Paglione, J. Strong surface scattering in ultrahigh-mobility $\mathrm{Bi}_{2} \mathrm{Se}_{3}$ topological insulator crystals. Phys. Rev. B 2010, 81, 241301. [CrossRef]

11. Ren, Z.; Taskin, A.; Sasaki, S.; Segawa, K.; Ando, Y. Large bulk resistivity and surface quantum oscillations in the topological insulator $\mathrm{Bi}_{2} \mathrm{Te}_{2}$ Se. Phys. Rev. B 2010, 82, 241306. [CrossRef]

12. Yoshimi, R.; Tsukazaki, A.; Kozuka, Y.; Falson, J.; Takahashi, K.; Checkelsky, J.; Nagaosa, N.; Kawasaki, M.; Tokura, Y. Quantum Hall effect on top and bottom surface states of topological insulator (Bi1-xSbx) ${ }_{2} \mathrm{Te}_{3}$ films. arXiv 2014, arXiv:1409.3326. [CrossRef]

13. Xia, B.; Ren, P.; Sulaev, A.; Liu, P.; Shen, S.Q.; Wang, L. Indications of surface-dominated transport in single crystalline nanoflake devices of topological insulator $\mathrm{Bi}_{1.5} \mathrm{Sb}_{0.5} \mathrm{Te}_{1.8} \mathrm{Se}_{1.2}$. Phys. Rev. B 2013, 87, 085442. [CrossRef]

14. Xu, Y.; Miotkowski, I.; Liu, C.; Tian, J.; Nam, H.; Alidoust, N.; Hu, J.; Shih, C.K.; Hasan, M.Z.; Chen, Y.P. Observation of topological surface state quantum Hall effect in an intrinsic three-dimensional topological insulator. Nat. Phys. 2014, 10, 956-963. [CrossRef] 
15. Neupane, M.; Xu, S.Y.; Wray, L.A.; Petersen, A.; Shankar, R.; Alidoust, N.; Liu, C.; Fedorov, A.; Ji, H.; Allred, J.M.; et al. Topological surface states and Dirac point tuning in ternary topological insulators. Phys. Rev. B 2012, 85, 235406. [CrossRef]

16. Arakane, T.; Sato, T.; Souma, S.; Kosaka, K.; Nakayama, K.; Komatsu, M.; Takahashi, T.; Ren, Z.; Segawa, K.; Ando, Y. Tunable Dirac cone in the topological insulator $\mathrm{Bi}_{2}-\mathrm{xSbxTe}-\mathrm{ySey}$. Nat. Commun. 2012, 3, 636. [CrossRef] [PubMed]

17. Chen, C.; Xie, Z.; Feng, Y.; Yi, H.; Liang, A.; He, S.; Mou, D.; He, J.; Peng, Y.; Liu, X.; et al. Tunable Dirac fermion dynamics in topological insulators. Sci. Rep. 2013, 3. [CrossRef] [PubMed]

18. Sterzi, A.; Manzoni, G.; Sbuelz, L.; Cilento, F.; Zacchigna, M.; Bugnon, P.; Magrez, A.; Berger, H.; Crepaldi, A.; Parmigiani, F. Bulk diffusive relaxation mechanisms in optically excited topological insulators. Phys. Rev. B 2017, 95, 115431. [CrossRef]

19. Pan, Z.H.; Fedorov, A.; Gardner, D.; Lee, Y.; Chu, S.; Valla, T. Measurement of an exceptionally weak electron-phonon coupling on the surface of the topological insulator $\mathrm{Bi}_{2} \mathrm{Se}_{3}$ using angle-resolved photoemission spectroscopy. Phys. Rev. Lett. 2012, 108, 187001. [CrossRef] [PubMed]

20. Damascelli, A.; Hussain, Z.; Shen, Z.X. Angle-resolved photoemission studies of the cuprate superconductors. Rev. Mod. Phys. 2003, 75, 473. [CrossRef]

21. Chen, Y.; Analytis, J.G.; Chu, J.H.; Liu, Z.; Mo, S.K.; Qi, X.L.; Zhang, H.; Lu, D.; Dai, X.; Fang, Z.; et al. Experimental realization of a three-dimensional topological insulator, $\mathrm{Bi}_{2} \mathrm{Te}_{3}$. Science 2009, 325, $178-181$. [CrossRef] [PubMed]

22. Sobota, J.A.; Yang, S.; Analytis, J.G.; Chen, Y.; Fisher, I.R.; Kirchmann, P.S.; Shen, Z.X. Ultrafast optical excitation of a persistent surface-state population in the topological insulator $\mathrm{Bi}_{2} \mathrm{Se}_{3}$. Phys. Rev. Lett. 2012, 108, 117403. [CrossRef] [PubMed]

23. Hajlaoui, M.; Papalazarou, E.; Mauchain, J.; Perfetti, L.; Taleb-Ibrahimi, A.; Navarin, F.; Monteverde, M.; Auban-Senzier, P.; Pasquier, C.; Moisan, N.; et al. Tuning a Schottky barrier in a photoexcited topological insulator with transient Dirac cone electron-hole asymmetry. Nat. Commun. 2014, 5, 3003. [CrossRef] [PubMed]

24. Crepaldi, A.; Ressel, B.; Cilento, F.; Zacchigna, M.; Grazioli, C.; Berger, H.; Bugnon, P.; Kern, K.; Grioni, M.; Parmigiani, F. Ultrafast photodoping and effective Fermi-Dirac distribution of the Dirac particles in $\mathrm{Bi}_{2} \mathrm{Se}_{3}$. Phys. Rev. B 2012, 86, 205133. [CrossRef]

25. Bugini, D.; Boschini, F.; Hedayat, H.; Yi, H.; Chen, C.; Zhou, X.; Manzoni, C.; Dallera, C.; Cerullo, G.; Carpene, E. Ultrafast spin-polarized electron dynamics in the unoccupied topological surface state of $\mathrm{Bi}_{2} \mathrm{Se}_{3}$. J. Phys. Condens. Matter 2017, 29, 30LT01. [CrossRef] [PubMed]

26. Hedayat, H.; Bugini, D.; Yi, H.; Chen, C.; Zhou, X.; Cerullo, G.; Dallera, C.; Carpene, E. Femtosecond dynamics of spin-polarized electrons in topological insulators. IEEE Magn. Lett. 2017. [CrossRef]

27. Zhu, S.; Ishida, Y.; Kuroda, K.; Sumida, K.; Ye, M.; Wang, J.; Pan, H.; Taniguchi, M.; Qiao, S.; Shin, S.; et al. Ultrafast electron dynamics at the Dirac node of the topological insulator Sb2Te3. Sci. Rep. 2015, 5, 13213. [CrossRef] [PubMed]

28. Chen, C.; He, S.; Weng, H.; Zhang, W.; Zhao, L.; Liu, H.; Jia, X.; Mou, D.; Liu, S.; He, J.; et al. Robustness of topological order and formation of quantum well states in topological insulators exposed to ambient environment. Proc. Natl. Acad. Sci. USA 2012, 109, 3694. [CrossRef] [PubMed]

29. Boschini, F.; Hedayat, H.; Dallera, C.; Farinello, P.; Manzoni, C.; Magrez, A.; Berger, H.; Cerullo, G.; Carpene, E. An innovative $\mathrm{Yb}$-based ultrafast deep ultraviolet source for time-resolved photoemission experiments. Rev. Sci. Instr. 2014, 85, 123903. [CrossRef] [PubMed]

30. Carpene, E.; Mancini, E.; Dallera, C.; Ghiringhelli, G.; Manzoni, C.; Cerullo, G.; De Silvestri, S. A versatile apparatus for time-resolved photoemission spectroscopy via femtosecond pump-probe experiments. Rev. Sci. Instr. 2009, 80, 055101. [CrossRef] [PubMed]

31. Hajlaoui, M.; Papalazarou, E.; Mauchain, J.; Lantz, G.; Moisan, N.; Boschetto, D.; Jiang, Z.; Miotkowski, I.; Chen, Y.; Taleb-Ibrahimi, A.; et al. Ultrafast surface carrier dynamics in the topological insulator $\mathrm{Bi}_{2} \mathrm{Te}_{3}$. Nano Lett. 2012, 12, 3532-3536. [CrossRef] [PubMed]

32. Sánchez-Barriga, J.; Battiato, M.; Krivenkov, M.; Golias, E.; Varykhalov, A.; Romualdi, A.; Yashina, L.; Minár, J.; Kornilov, O.; Ebert, H.; et al. Subpicosecond spin dynamics of excited states in the topological insulator $\mathrm{Bi}_{2} \mathrm{Te}_{3}$. Phys. Rev. B 2017, 95, 125405. [CrossRef] 
33. Neupane, M.; Xu, S.Y.; Ishida, Y.; Jia, S.; Fregoso, B.M.; Liu, C.; Belopolski, I.; Bian, G.; Alidoust, N.; Durakiewicz, T.; et al. Gigantic surface lifetime of an intrinsic topological insulator. Phys. Rev. Lett. 2015, 115, 116801. [CrossRef] [PubMed]

34. Cacho, C.; Crepaldi, A.; Battiato, M.; Braun, J.; Cilento, F.; Zacchigna, M.; Richter, M.; Heckmann, O.; Springate, E.; Liu, Y.; et al. Momentum-resolved spin dynamics of bulk and surface excited states in the topological insulator $\mathrm{Bi}_{2} \mathrm{Se}_{3}$. Phys. Rev. Lett. 2015, 114, 097401. [CrossRef] [PubMed]

35. Jozwiak, C.; Sobota, J.A.; Gotlieb, K.; Kemper, A.F.; Rotundu, C.R.; Birgeneau, R.J.; Hussain, Z.; Lee, D.H.; Shen, Z.X.; Lanzara, A. Spin-polarized surface resonances accompanying topological surface state formation. Nat. Commun. 2016, 7, 13143. [CrossRef] [PubMed]

36. Sumida, K.; Ishida, I.; Zhu, S.; Ye, M.; Pertsova, A.; Triola, C.; Kokh, K.A.; Tereshchenko, O.E.; Balatsky, A.V.; Shin, S.; Kimura, A. Prolonged duration of nonequilibrated Dirac fermion in neutral topological insulators. Sci. Rep. 2017, 7, 14080. [CrossRef] [PubMed]

(c) 2018 by the authors. Licensee MDPI, Basel, Switzerland. This article is an open access article distributed under the terms and conditions of the Creative Commons Attribution (CC BY) license (http://creativecommons.org/licenses/by/4.0/). 